\title{
Impairment of Calcium-dependent inactivation of TRPC6 Mediated by Calmodulin Underlies Renal Channelopathy
}

\author{
Onur K Polat ${ }^{1}$, Masayuki X Mori ${ }^{1}$, Masatoshi Uno ${ }^{2}$, Hidehito Tochio ${ }^{2}$, Yasuo Mori ${ }^{1}$ \\ ${ }^{1}$ Graduate School of Engineering, Department of Synthetic and Biological Chemistry, Japan, ${ }^{2}$ Kyoto University, \\ Graduate School of Science, Department of Biophysics, Japan
}

TRPC6 channel activity is suppressed by the intracellular $\mathrm{Ca}^{2+}$ through a negative feedback regulation known as $\mathrm{Ca}^{2+}$ dependent inactivation (CDI), in which calmodulin ( $\mathrm{CaM}$ ) has been proposed to play a critical role. Here, our molecular mutations and dissections with functional and biophysical analysis have revealed new detail that equal contribution of $\mathrm{N}$ and C-lobes of CaM to CDI and which could be accomplished by $\mathrm{Ca}^{2+}$-dependent CaM binding to the TRPC6 CaMbinding domain (CBD) in a 1:2 stoichiometry. This ensemble prerequisite was arranged via assembly of the coiled-coil segment adjacent to the CBD. The deletion of this segment markedly decelerated CDI of TRPC6 currents, suggesting that $\mathrm{CDI}$ is induced by bridging of two CBDs by a single CaM molecule. Naturally occurring gain-of-function TRPC6 mutations are involved in autosomal dominant focal segmental glomerulosclerosis (FSGS). Surprisingly, the FSGSTRPC6 mutations in the coiled-coil severely hamper CDI and induce "rim-like" morphological changes in podocytes. Our study provides novel structural insights into the CDI of TRPC6 channel, and the disruption of CDI leads to the pathophysiological significance in kidney disease. 